\title{
Utilisation d'une technique journalistique dans l'apprentissage de l'entretien médical « Une expérience développée dans le cursus préclinique de la Faculté de Médecine Xavier Bichat »
}

\author{
Françoise BLANCHET ${ }^{*}$, Daniel RAICHVARG** , Dominique MAILLARD***
}

\begin{abstract}
Résumé But: Ce travail décrit une formation mise en place à la faculté de médecine Xavier Bichat dans le cursus préclinique des études médicales. Elle a pour but de développer les capacités de communication des étudiants, en utilisant une technique journalistique : le "deux minutes radio ". Méthodes : Les étudiants ont été répartis en 5 groupes de 20 pendant 2 demi-journée de 3 heures. Cinq textes sur la douleur leur ont été proposés. Cette formation s'est déroulée en quatre étapes : 1-apprentissage de la technique de transformation d'un texte-source en une communication radiophonique de vulgarisation; 2-travail d'analyse et transformation du texte ; 3- présentation orale au groupe d'une durée de 2 minutes. Résultats : La performance globale des orateurs (score maximal de 5) évaluée par les enseignants et les étudiants (Q1-Q3) concerne la cohérence du contenu du "deux minutes radio " et l'effet suscité par l'exposé sur le groupe ; les scores sont respectivement de 3,91+0,11 et de 4,39+0,07. Il existe une corrélation significative entre l'évaluation des enseignants et celle des étudiants $(p<0,0001)$. Un score moyen de 3,76 + 0,24 rend compte de l'évaluation par les enseignants du respect de la technique de communication. Conclusions : Les étudiants ont acquis par cette technique des aptitudes à délivrer un message concis et clair les préparant à la pratique de l'entretien médical. Cette formation peut être reproduite facilement sur d'autres sites.

Mots clés Etudiants; entretien médical; apprentissage de la communication orale.

Summary Aim: This study describes a training offered in the pre-clinical course of the medical curriculum of the faculté de médecine Xavier Bichat. Its goal is to develop communication skills of the students by using a journalistic technique: the "two minutes-radio ". Methods: The students were distributed into five groups of 20 each, for a duration of two half-days of 3 hours each. Five texts about "pain " were studied. The training was a four stages process: 1-learning the technique of transforming an original text into a short radio popular style communication; 2analysing and transforming the text ; 3- a two-minute oral communication to the group of students. Results: the overall performance of the speakers (maximal score offive), scored by the teachers and the students (questions 1-3), assess the content of the " two-minutes radio " communication and the kind of interaction they created with the group. Mean score were respectively: $3.91+0.11$ and $4.39+0.07$. There was a significant correlation between the two evaluations of teachers and students $(p<0.0001)$. A mean score of $3.76+0.24$ given by the teachers to the questions Q4-1 to Q4-4, measured the appropriate use of the technique of communication. Conclusion: this training allows the students to deliver concise and clear messages and prepares them to the practice of a medical interview. This training can be easily reproduced in other medical schools.
\end{abstract}

Key Words Students; medical interview ; oral communication learning.

Pédagogie Médicale $2001 ; 2: 87-92$.

* Département de Sciences Humaines - Faculté de Médecine Xavier Bichat (Paris 7)

${ }^{*}$ *Centre de recherche sur la culture et les musées - Université de Bourgogne (Dijon)

* * Département de Pédagogie - Faculté de Médecine Xavier Bichat (Paris 7)

Correspondance : Dr Dominique Maillard - Hôpital Louis Mourier - Explorations Fonctionnelles - 178, rue des Renouillers 92700 Colombes - France - Tél. : 33 (0)1476062 49 - Télécopie : 33 (0)1 47606269

Couriel : dominique.maillard@mr.ap-hop-paris.fr 


\section{Concepts et Innovations}

\section{Introduction}

L'importance d'une formation de l'étudiant en médecine aux techniques de communication utilisées dans l'entretien médical est reconnue par de nombreuses facultés et équipes de recherche ${ }^{1-5}$. Un entretien de qualité permet, en effet, au médecin d'exercer son raisonnement clinique et d'obtenir des informations pertinentes lui permettant de parvenir à des hypothèses diagnostiques fiables et valides et à des choix thérapeutiques mieux adaptés au patient ${ }^{6,7}$. Par ailleurs, le satisfaction du patient, la perception qu'il peut avoir de la compétence du médecin et son investissement actif dans les soins sont très fortement corrélées aux compétences communicationnelles du médecin ${ }^{8,9}$. La qualité de l'entretien dépend de l'habileté du médecin à créer un climat de confiance, de ses capacités d'écoute et de son aptitude à identifier les messages contenus dans le discours du patient. Reformuler, clarifier, synthéser les propos du patient traduisent la volonté et la capacité du médecin à le comprendre et caractérisent son attitude empathique ${ }^{10}$.

Ce travail décrit une formation mise en place à la faculté Xavier Bichat (Paris 7) dans le cursus préclinique dès la deuxième année des études médicales. Elle a pour but d'améliorer les capacités de communication des étudiants. Plus spécifiquement, cette formation cherche à développer chez l'étudiant des capacités d'expression orale à partir de l'analyse et de la synthèse d'un texte scientifique, en utilisant une technique journalistique, le " deux minutes radio ». Elle permet d'entraîner les étudiants à délivrer un message concis et clair et à les préparer à la pratique de l'entretien médical. Cet enseignement s'inscrit dans la continuité de l'enseignement de Sciences Humaines de la première année où les objectifs pédagogiques sont centrés sur le développement des capacités d'analyse et de synthèse à partir de commentaires de textes d'histoire des Sciences et de la Médecine.

\section{Méthodes}

\section{2-1. Contenu de la formation}

L'enseignement a été réalisé par cinq enseignants en Sciences de la Communication, journalistes professionnels dans des revues scientifiques. La cohorte choisie était constituée par l'ensemble des 102 étudiants de deuxième année, répartis en cinq groupes de 20. La formation a été organisée sur deux demi-journées consécutives de 3 heures. La première demi-journée a consisté à apprendre la technique de communication orale ; la seconde a été consacrée à la réalisation de l'exposé oral.
Le déroulement de la première demi-journée s'est réalisé en trois étapes : 1-apprentissage de la technique de transformation d'un texte-source en une communication radiophonique de vulgarisation ; 2 - distribution d'un texte scientifique aux étudiants regroupés en binomes ; 3- travail d'analyse et transformation du texte choisi.

L'activité de la deuxième demi-journée a permis de compléter le travail d'analyse et de préparer le texte de vulgarisation en vue de sa présentation orale devant le groupe sous forme d'un "deux minutes radio ». L'apprentissage de la technique journalistique a été découverte par l'étudiant en début de journée dans un travail de groupe interactif, à partir de deux communications de vulgarisation sélectionnées pour leur qualité didactique. Les techniques journalistiques de transformation du texte-source en une communication de vulgarisation de deux minutes comprennent : l'analyse du contenu scientifique, du point de vue de sa valeur intrinsèque et de son adéquation au destinataire ; la recherche des étapes du déroulement argumentatif ; les choix faits pour l'accroche et la conclusion ; les formes d'interpellation du destinataire (intégration du questionnement du destinataire, formes de relance de l'attention...) ; la sélection et l'analyse de la valeur des images et les métaphores.

Les textes, choisis par l'équipe enseignante, abordaient la douleur sous différentes approches ( $c$. annexe 1, page 90). Ils ont été proposés à chaque groupe d'étudiants. A l'intérieur de chaque groupe, un texte a été distribué à chaque binome.

\section{2-2. Evaluation des acquisitions}

L'acquisition des objectifs a été appréciée sur trois points : 1- le respect de la technique de communication (existence d'une phrase d'accroche, étapes du déroulement argumentatif, utilisation de métaphores, conclusion) ; 2- la cohérence du contenu du "deux minutes radio » avec le document source (présentation compréhensible, identification des messages du texte-source, envie d'acquérir d'autres connaissances) ; 3- l'effet suscité par l'exposé sur le groupe d'étudiants (compréhension facile, reformulation aisée des messages du texte-source, envie d'acquérir d'autres connaissances sur le sujet).

La performance de l'orateur a été quantifiée à la fois par l'enseignant et chaque étudiant du groupe lors de la présentation selon les grilles d'évaluation présentées en annexe, page 90. Les règles de l'évaluation ont été présentées préalablement aux étudiants au début de la 


\begin{tabular}{|l|c|c|c|}
\hline \multicolumn{3}{|c|}{ Tableau 1 : Evaluation comparée de la performances des étudiants } \\
\hline Résultats & $\begin{array}{c}\text { Scores donnés par } \\
\text { les enseignants }\end{array}$ & $\begin{array}{c}\text { Scores donnés par } \\
\text { les étudiants }\end{array}$ & Différence \\
\hline Score global & $3,91(0,11)$ & $4,39(0,07)$ & $\mathrm{S}(\mathrm{p}<0,0001)$ \\
Q1 & $3,90(0,19)$ & $\mathrm{S}(\mathrm{p}<0,0001$ \\
Q2 & $3,81(0,18)$ & $\mathrm{S}(\mathrm{p}<0,0001)$ \\
Q3 & $4,02(0,22)$ & $4,39(0,11)$ & $\mathrm{S}(\mathrm{p}=0,017)$ \\
Q4-1 & $4,06(0,20)$ & \\
Q4-2 & $3,67(0,25)$ & & \\
Q4-3 & $3,30(0,25)$ & & \\
Q4-4 & $3,90(0,19)$ & & \\
\hline
\end{tabular}

formation et ont été rappelées au moment de la distribution des grilles (début des présentations orales). Létudiant s'est autoévalué à la fin de l'exposé oral et a exprimé les difficultés rencontrées au cours de la réalisation de la note de synthèse. Par ailleurs, les commentaires et les questions du groupe d'étudiants envers l'orateur permettaient à l'étudiant de percevoir l'effet de son exposé sur l'auditoire.

\section{2-3. Statistiques}

Les résultats sont exprimés par leur valeur moyenne +2 erreurs standard à la moyenne. Les comparaisons de notations entre étudiants et enseignants ont été faites par test de T apparié de Student. Le degré de relation entre deux variables a été déterminé par le calcul du coefficient de corrélation. Une différence significative est acceptée pour une valeur de $\mathrm{p}$ inférieure à 0.05 .

\section{Résultats}

Tous les étudiants présents ont participé à l'évaluation. Le tableau 1 résume les scores moyens attribuées lors de l'éva- luation par les enseignants et les étudiants. La performance globale de l'orateur (cohérence du deux minutes radio, effets suscités par l'exposé sur le groupe) mesurée par les questions Q1 à Q3 est considérée comme bonne tant par les enseignants que par l'auditoire d'étudiants : 3,91+0,11 et 4,39+0,07 pour un score maximal de 5. L'existence d'une corrélation significative $(p<0,0001)$ entre les deux séries de scores, donnés d'une part, par les enseignants et d'autre part, par les étudiants, valide l'hypothèse du respect des règles d'évaluation par les étudiants. Cependant le score attribué par les étudiants à leurs camarades est significativement plus élevé comparativement à celui des enseignants $(p<0,0001)$, ce qui traduit une évaluation de la part des étudiants fiable mais plus généreuse. Cette différence de comportement évaluatif entre enseignants et étudiants est retrouvée systématiquement pour chaque question Q1, Q2 et Q3.

Enfin la technique de communication, évaluée par les questions Q4-1 à Q4-4 est bien respectée par les étudiants, comme en témoigne le score global moyen rapporté par les enseignants : 3,76+0,24 (score maximal de 5). 


\section{Concepts et Innovations}

\section{Annexe 2}

\section{Grille 1 : Evaluation de la performance de l'orateur par l'enseignant}

Nous voulons connaître votre évaluation de l'étudiant en utilisant le code suivant :
1. Performance nulle
2. Performance passable
4. Bonne performance
5. Très bonne performance
3. Performance moyenne
Nom :
Prénom :

Q1 La présentation de l'étudiant était très compréhensible.

$\begin{array}{lllll}1 & 2 & 3 & 4 & 5 \\ 1 & 2 & 3 & 4 & 5 \\ 1 & 2 & 3 & 4 & 5\end{array}$

Létudiant a utilisé les règles de la technique de présentation orale avec:
Q4-1 l'existence d'une phrase d'accroche.
$\begin{array}{lllll}1 & 2 & 3 & 4 & 5\end{array}$
Q4-2 les étapes du déroulement argumentatif.
$\begin{array}{lllll}1 & 2 & 3 & 4 & 5\end{array}$
Q4-3 l'utilisation de métaphores.
$\begin{array}{lllll}1 & 2 & 3 & 4 & 5\end{array}$
Q4-4 une conclusion.

$\begin{array}{lllll}1 & 2 & 3 & 4 & 5\end{array}$

\section{Grille 2 : Evaluation de la performance de l'orateur par les étudiants}

Votre évaluation de la performance de votre camarade est utile pour l'aider à s'améliorer.

Soyez le plus honnête possible. Nous voulons connaître votre évaluation en utilisant le code suivant :
1. Je suis totalement en désaccord
2. Je suis en désaccord
3. Je n'ai pas d'opinion
4. Je suis en accord
5. Je suis parfaitement en accord

Nom :

Prénom :

Q1 La présentation de mon collègue était très compréhensible.

Q2 Je serais capable de reformuler les messages du texte source.

Q3 A la suite de cette présentation, j'ai envie d'acquérir d'autres connaissances sur ce sujet. 


\section{Utilisation d'une technique journalistique...}

\section{Discussion}

Cette première évaluation des acquisitions par les étudiants est satisfaisante dans le court terme. Il est important de noter que cette formation délivrée à un stade préclinique du cursus des études médicales rend compte, malgré sa courte durée, de l'entrainement de l'étudiant à identifier des messages, à les hiérarchiser puis à les reformuler dans un langage simple et compréhensible par le plus grand nombre. Cette méthode ludique semble très motivante pour les étudiants de deuxième année et ceuxci réussissent assez bien à transformer les items scientifiques des textes en langage radiophonique ( $c f$. encadré cicontre). De surcroît, l'évaluation en retour par leurs camarades permet à l'orateur de vérifier la compréhension de son message. Cette situation sera retrouvée en clinique lors de l'entretien médical où le médecin devra vérifier la compréhension de son message auprès du malade. Le texte, en se substituant au discours du patient, permet d'acquérir des compétences communicationnelles de base nécessaires au dialogue entre le malade et son médecin. Néanmoins, cette acquisition à court terme des techniques de communication mérite d'être réévaluée lors de la première année du cursus clinique quand les étudiants débuteront leur expérience hospitalière auprès du malade. En effet, la littérature est pessimiste sur la persistance de ces aptitudes qui semblent se détériorer pendant les études médicales. Les étudiants apprennent à reconstituer l'histoire de la maladie et à résoudre des problèmes de plus en plus complexes aux dépens de l'intégration de la maladie chez un malade singulier et de ce fait leurs comportements empathiques diminuent ${ }^{11-14}$. La situation d'incertitude créée par la complexité de la pathologie et la nécessaire objectivation de la maladie leur font perdre de vue l'importance de la relation avec le patient dans leur décision diagnostique ou thérapeutique. Craig ${ }^{14}$ conclut à la nécessité d'une formation, répartie pendant tout le cursus des études médicales, permettant à l'étudiant de consolider les acquisitions de la phase préclinique et de les intégrer à sa future pratique professionnelle.

Dans notre faculté, cet apprentissage de la reformulation est réitéré au cours de cette même deuxième année préclinique ; les étudiants s'entraînent cette fois, à partir d'un film, à reformuler les dialogues entre un malade et différents médecins rencontrés lors de consultations successives. Cette deuxième formation fait suite au " deux minutes radio » et met graduellement l'étudiant en situation pratique en permettant d'apprécier son aptitude à analyser le discours et le comportement du malade.

\section{Un exemple de « deux minutes radio »}

\author{
Rédigés par un étudiant de deuxième année \\ à partir du texte d'Alain Eschalier : \\ "Les antidépresseurs contre la douleur" \\ publié dans : Pour la science - juin 1994 : 36-42.
}

Maux de tête, mal de dos, rhumatismes, douleurs cancéreuses sont aujourd'hui soulagés par les antidépresseurs $(\mathrm{AD})$. En effet, l'aspirine, le paracétamol, la morphine qui sont les trois seuls antalgiques couramment utilisés, restent parfois impuissants face à ce type de douleurs. Le recours aux $\mathrm{AD}$ est alors à envisager.

Il a été démontré que les $\mathrm{AD}$ n'avaient pas seulement une action sur les états dépressifs proprement dits mais également sur les douleurs chroniques. Ceci a été d'abord expérimenté sur les animaux : « si votre chien a mal à la patte, donnez-lui de l'aspirine, s'il continue à boiter, changez pour du Prozac et il retrouvera son allure de grand chasseur ». Puis on s'est rendu compte que les patients traités par un $\mathrm{AD}$ étaient plus soulagés que ceux traités par simple placebo.

Une question se pose toutefois : les AD agissent-ils directement sur la douleur ou améliorent-ils l'humeur du malade qui supporte alors mieux sa douleur ? Les médecins semblent croire qu'il existe réellement un effet antalgique des $\mathrm{AD}$, ce qui justifie leur prescription pour apaiser certaines douleurs. En effet, ils agissent en inhibant l'émission des messages douloureux véhiculés par influs nerveux. Mais attention, là aussi les effets sont limités. Par exemple, pour les migraines, ils espacent les crises, diminuent leur intensité et leur durée, mais pourtant un malheureux sur cinq ne verra pas ses migraines diminuer par la prise d'AD.

En bref, les $\mathrm{AD}$ semblent être une révolution dans le soulagement des douleurs récalcitrantes. Toutefois pour les quelques résistants à cette thérapie, il faudra envisager d'autres traitements.

Affaire à suivre... 


\section{Concepts et Innovations}

\section{Conclusions}

Ce modèle de communication journalistique (le « deux minutes radio ") appliqué à l'analyse et la synthèse d'un texte scientifique permet de développer chez l'étudiant en médecine, au niveau préclinique, des capacités d'expression orale. Cette formation, par l'intermédiaire d'enseignants formés en sciences de la communication, les entraîne à délivrer un message concis et clair et les prépare à la pratique de l'entretien médical. Elle a l'avantage d'utiliser des techniques simples et peu coûteuses et peut être reproduite dans toute faculté de médecine qui en perçoit le besoin.

\section{Annexe 1 : Références des textes}

1. Ronald Melzack. Les membres fantômes. Pour la science $1992 ; 176: 48-54$.

2. Alain Eschalier. Les antidépresseurs contre la douleur. Pour la science 1994 ; 200 : 36-42.

3. Jean Bruxelle. L'effet placebo dans le traitement de la douleur 1994 ; 44 : 1919-1922.
4. Louis Brasseur. Réflexions sur la prescription de morphiniques pour traiter les douleurs chroniques d'origine non cancéreuse. La lettre de l'institut UPSA de la douleur 1998 ; $6: 1-7$.

5. Daniel Annequin. La douleur de l'enfant : une reconnaissance tardive. La recherche 2000 ; 336 : $42-47$.

\section{Références}

1. Students II-evaluation of a training program. 1976 ; Lancet : 558-60.

2. Maguire GP, Clarke D, Jolley B. An experimental comparison of three courses in history-taking skills for medical student. Medical Education 1977; 11 : $175-82$

3. Evans BJ, Stanley RO, Mestrovic R, Rose L. Effects of communication skills on students' diagnostic efficiency. Medical Education 1991 ; 25 : 517-26.

4. Novack DH, Volk G, Droosman DA, Lipkin M Jr. Medical interviewing and interpersonal skills teaching in US medical schools: progress, problems, and promise. JAMA 1993 ; 269 : 2101-05.

5. Kaplan CB, Siegel B, Madill JM, Epstein RM. Communication and the medical interview - strategies for learning and teaching. J Gen. Intern. Med. 1997 ; 12 (issue 2 Suppl) : S49-S55.

6. Alroy $G$, Ber R, Kramer D. An evaluation of the short-term effects of an interpersonal skills course. Medical Education 1984 ; 18 : 85-89.

7. Carmel S, Bernstein J. Identifying with the patients : an intensive programme for medical students. Medical Education 1986 ; 20 : 432-36.

8. Feletti G, Firman D, Sanson-Fisher R. Patient satisfaction with primary-care consultations. J Behav Med 1986 ; 9 : 389-99.
9. ampton JR, Harrison MJG, Mitchell JRA, Richard $J S$, Semour C. Relative contributions of historytaking, physical examination, and laboratory investigation to diagnosis and management of medical out-patients. BMJ 1975 ; 2 : 486-89.

10. Des Marchais JE, Jean P, Castonguay LG. Training psychiatrics and family doctors in evaluating interpersonnal skills. Medical Education 1990 ; 24 : 376-81.

11. Kauss DR, Robbins AS, Abrass I, Bakaitis RF, Anderson $L A$. The long-term effectiveness of interpersonal skills training in medical school. J Med Ed $1980 ; 55: 595-601$.

12. Engler CM, Saltzman GA, Walker ML, Wolf FM. Medical student acquisition and retention of communication and interviewing skills. J Med Ed $1981 ; 56$ : 572-79.

13. Kraan HF, Cringen AAM, De Vriwe $M W$, Zuidweg J, Imbos J, Van Der Vleuten CP. To what extent are medical interviewing skills teachable? Med Teach $1990 ; 12: 315-28$.

14. Craig JL. Retention of interviewing skills learned by first-year medical students : a longitudinal study. Med Ed 1992 ; 26 : 276-81. 